\title{
SESSION 8: Fatty acid and vitamin transport
}

\section{Bicarbonate dependence of basolateral SCFA transport in the rumen}

\author{
Franziska Dengler, Reiko Rackwitz, Anke Schmidt-Mähne, Gotthold Gäbel
}

Institute for Veterinary Physiology,An den Tierkliniken 7, 04103 Leipzig, email:dengler@vmf.uni-leipzig.de

Introduction: Permeation of SCFA by undissociated diffusion as well as by a SCFA-bicarbonateexchange could be detected on the apical side of sheep rumen epithelium. However, transport across the basolateral membrane is still unsettled. Dudeja and Ramaswamy (Physiology of the Gastrointestinal Tract 2006, p.1881) suggested a SCFA-bicarbonate-exchange on the basolateral side of colonocytes as well and postulated it to be a monocarboxylate transporter (MCT).

Aim: to elucidate the relevancy of bicarbonate for basolateral SCFA-transport and the corresponding transport proteins.

Methods: Sheep rumen epithelium obtained from the ventral sac was mounted in Ussing chambers in the presence or absence of $20 \mathrm{mM}$ bicarbonate (bic) with a SCFA-concentration of $10 \mathrm{mM}$ acetate (ac) and butyrate (bu) each, partially labelled radioactively. After 120 minutes incubation, the epithelia were washed thrice and the buffer solution was replaced by a SCFA-free buffer solution. Thereafter efflux of labelled SCFA out of the epithelium was measured in buffer samples taken at certain time intervals. In a second approach, we implied a concentration gradient of bic from the serosal $(90 \mathrm{mM})$ to mucosal (20mM) side and studied fluxes with ac and bu from the mucosal to the serosal side. We tried to block the efflux by adding inhibitors of MCT (pHMB or phloretin) to the serosal side.

Results: The efflux of SCFA showed a considerable discrepancy between the bic-free and -containing approach. On both the mucosal and serosal side there was a nearly fourfold increase of SCFA efflux with bic present. Independent from the presence of bic, there were side dependent differences between ac and bu efflux. In contrast to the mucosal side, where efflux of ac was quantitatively similar to bu efflux, on the serosal side bu efflux was about twice that of ac, whereat these proportions were maintained in the presence of bic. Applying a bic concentration gradient, we observed an increase in ms-flux rates of about $50 \%$ compared to fluxes without a gradient. Addition of $\mathrm{pHMB}$ or phloretin, respectively, induced only small changes of both ac/bu effluxes and ms transport rates.

Conclusions: Our data clearly show, that there are different mechanisms on the apical and basolateral membrane for transport of SCFA and, at least on the basolateral side, there seem to be different transport characteristics or even mechanisms for ac and bu. One of these mechanisms is obviously coupled to bic. However, we could not definitely prove it to be a MCT so far.

This study is supported by DFG (GA329/7-1) 


\title{
A co-culture system of human intestinal Caco-2/TC7 and hepatic cell lines: investigations on beta-carotene and retinol transport and metabolism
}

\author{
Carlotta Rossi, Barbara Guantario, Diana Bellovino, Simonetta Ferruzza, Maria Laura \\ Scarino, Christiane Guguen-Guillouzo* and Yula Sambuy \\ National Research Institute on Food and Nutrition, Rome, Italy; *INSERM U522, IFR 140, Université de Rennes1, Rennes, \\ France
}

Introduction: vitamin A (all-trans retinol) and its metabolites (retinoids) exert many important and diverse functions in cells, most notably in vision, cell proliferation and differentiation, immune function, neural function, and early embryonic development. Retinoids act primarily by regulating gene expression. Vitamin A is absorbed from animal food sources and/or derived from $\beta$-carotene, which is found in plants. Dietary retinoid transport and metabolism involves the interplay between different tissues, primarily the intestine for transport and metabolism by differentiated enterocytes and the liver for secondary metabolism at the level of the hepatocytes.

Aim: to reproduce in vitro retinoids transport and metabolism between the intestine and the liver, by co-culturing differentiated human intestinal Caco-2/TC7 cells with two hepatic cell lines, murine 3A cells and the more differentiated human HepaRG cells.

Methods: in co-culture experiments, Caco-2/TC7 were grown and differentiated on filter culture inserts for three weeks, then transferred to culture wells with $3 \mathrm{~A}$ or HepaRG cells seeded on the bottom and maintained in co-culture for further 24 hours. During the co-culture period $\beta$-carotene or retinol were added to the apical side of the intestinal cells. Functionality of the co-culture model was assayed using different parameters: analysis of Caco-2/TC7 monolayer integrity, expression of specific genes involved in retinol metabolism (BCMO1, CYP26A1), and the retinol-dependent secretion of RBP4 from the hepatocytes. Even though RBP4 gene transcription is not affected by retinol availability, retinol is essential for a regular secretion of this protein from the cell.

Results: we found that retinol or $\beta$-carotene added to the apical medium of $\mathrm{Caco} 2 / \mathrm{TC} 7$ cells were transported, partially metabolised to retinol and retinol esters and secreted into the basolateral medium in contact with the hepatocytes, thus stimulating secretion of RBP4 from the latter.

Conclusion: this in vitro model can be considered a promising tool to analyze the absorption and metabolism of retinoids and could further be extended to investigations on other dietary compounds and molecules of pharmacological interest.

Work supported by EU FP6 Project LIINTOP Contract nuber STREP-037499 and by grant NUME (DM 3688/7303/08) from the Italian Ministry of Agriculture, Food \& Forestry (MiPAAF) 